\title{
When does the screening effect not hold?
}

\author{
Michael L. Stein \\ Department of Statistics, University of Chicago, Chicago, IL 60637, (E-mail: \\ stein@galton.uchicago.edu).
}

\begin{abstract}
The screening effect is the phenomenon of nearby observations yielding a good approximation to the optimal linear predictor of a spatial process based on a large set of observations. In addition to its obvious relevance to computation with large spatial datasets, knowing when a screening effect occurs is key to understanding the behavior of spatial processes. This work provides the first general results showing when an asymptotic screening effect does not hold by considering the prediction of a weakly stationary, isotropic process on $\mathbb{R}^{d}$ at a single location based on observing the process everywhere on $\mathbb{R}^{d}$ with white noise and letting the variance of the white noise tend to 0 . The main result shows that a screening effect does not hold if the isotropic spectral density fluctuates too much at high frequencies.
\end{abstract}

Keywords: Kriging; filtering; Gaussian process; isotropic process; screen effect

\section{Introduction}

For a random field $Z$ on $\mathbb{R}^{d}$ with finite second moments, consider predicting $Z$ at an unobserved location $x_{0}$ based on some set of observations $Z\left(x_{1}\right), \ldots, Z\left(x_{n}\right)$ via optimal (minimum mean squared error) linear interpolation, also known as kriging. One might generally expect this optimal linear interpolant to depend mainly on the behavior of $Z$ over distances not much larger than those from $x_{0}$ to the nearest observations to $x_{0}$. There is a number of ways that one could formalize this concept. One is to compare the 
mean squared prediction errors of the optimal predictor based on some subset of the observations near $x_{0}$ and the optimal predictor based on all of the observations. A closely related formalization is to consider to what extent the behavior of the optimal predictor can be determined just by knowing the behavior of the covariance function in some neighborhood of $x_{0}$. Both approaches are used in this paper.

The phenomenon of obtaining nearly optimal predictors by just using nearest neighbors to $x_{0}$ is known as the screening (or screen) effect in the geostatistical literature (Journel and Huijbregts, 1978 and Chilès and Delfiner, 2012). Especially for isotropic processes in more than one dimension, exact screening effects, in which distant observations have no effect on the optimal linear predictor, generally do not occur. Thus, it is natural to consider circumstances under which a screening effect holds asymptotically in some limit. Using $\epsilon$ to indicate a parameter controlling the observation locations, following the notation in Stein (2012), suppose $x_{0}=0$ and write $N_{\epsilon}$ for some set of observations near 0 and $F_{\epsilon}$ another set of observations generally more distant from 0 . Write $e(S)$ for the error of the optimal linear predictor of $Z(0)$ based on observing $Z$ on the set $S$. Then we will say an asymptotic screening effect holds if

$$
\lim _{\epsilon \downarrow 0} \frac{E e\left(N_{\epsilon} \cup F_{\epsilon}\right)^{2}}{E e\left(N_{\epsilon}\right)^{2}}=1
$$

In some settings, it will be convenient to define $N_{\epsilon} \cup F_{\epsilon}$ directly and call it $U_{\epsilon}$. In Stein (2012), $N_{\epsilon}=\left\{\epsilon x_{1}, \ldots \epsilon x_{n}\right\}$ and $F_{\epsilon}=\left\{y_{0}+\epsilon y_{1}, \ldots, y_{0}+\epsilon y_{m}\right\}$, where $x_{1}, \ldots, x_{n}$ are fixed (not depending on $\epsilon$ ), distinct, nonzero points in $\mathbb{R}^{d}, y_{0} \neq 0$ and $y_{1}, \ldots, y_{m}$ are fixed, distinct points in $\mathbb{R}^{d}$. It is possible to let $N_{\epsilon}$ be a set of fixed size in this setting because the points in $F_{\epsilon}$ are, for small $\epsilon$, all near $y_{0} \neq 0$ and thus are well-separated from 0 . If we do not want to allow any clear separation between the points in $N_{\epsilon}$ and those in $F_{\epsilon}$, then we will generally need to allow the number of points in $N_{\epsilon}$ to tend to $\infty$ as $\epsilon \downarrow 0$ in order for (1) to hold. For example, in Stein (2002), $U_{\epsilon}$ is the infinite lattice $\epsilon\left(y_{0}+j\right)$ for $j \in \mathbb{Z}^{d}, y_{0}$ a fixed point not in $\mathbb{Z}^{d}$ and $N_{\epsilon}$ is the intersection of 
this infinite lattice with $r_{\epsilon} B$, where $r_{\epsilon} / \epsilon \rightarrow \infty$ as $\epsilon \downarrow 0$ and $B \subset \mathbb{R}^{d}$ contains a neighborhood of the origin. The condition on $r_{\epsilon}$ guarantees that the number of points in $N_{\epsilon}$ tends to infinity as $\epsilon \downarrow 0$. Letting the number of nearby points grow without bound may not be what some have in mind when they think about the screening effect, but for $r_{\epsilon} / \epsilon$ bounded, I am unaware of any isotropic model in more than one dimension other than a pure nugget effect for which (1) will hold for this setting.

For a weakly stationary process $Z$ on $\mathbb{R}^{d}$ with spectral density $f$,

$$
\operatorname{cov}\{Z(x+h), Z(x)\}=\int_{\mathbb{R}^{d}} e^{i \omega^{T} h} f(\omega) d \omega
$$

for all $h$ and $x$. Stein $(2002,2011)$ studies conditions on $f$ under which a screening effect holds. For example, Stein (2011) assumes, among other conditions on $f$, that

$$
\lim _{|\omega| \rightarrow \infty} \sup _{|\nu|<R}\left|\frac{f(\omega+\nu)}{f(\omega)}-1\right|=0,
$$

which says that at high frequencies, the spectral density changes relatively negligibly when the frequency is changed by a modest amount. These works give examples showing that if some constraint is not put on the high frequency behavior of $f$, then a screening effect may not hold. In these situations, it is often the case that the corresponding autocovariance function possesses some lack of smoothness away from the origin. There are no previous results giving anything like inverses that say if $f$ does not obey a condition such as (2) then a screening effect does not hold.

The rest of this work only considers isotropic processes on $\mathbb{R}^{d}$, in which case the spectral density $f(\omega)$ depends only on $|\omega|$. Writing $f(u)$ for $u \geq 0$ as this isotropic spectral density, (2) is still applicable with $\omega$ and $\omega+\nu$ as positive reals. The symbol ^ over a function will be used to indicate a Fourier transform. Specifically, for a measurable function $\beta$ on $[0, \infty)$ satisfying $\int_{0}^{\infty}|\beta(u)| u^{d-1} d u<\infty$, the function of $x$ on $\mathbb{R}^{d}$ given by $\int_{\mathbb{R}^{d}} e^{i \omega^{T} x} \beta(|\omega|) d \omega$ depends only on $r=|x|$ and write $\widehat{\beta}(r)$ for this function on $[0, \infty)$. If, in 
addition, $\beta$ is nonnegative, then $\beta$ is a valid isotropic spectral density for a process on $\mathbb{R}^{d}$ and $\widehat{\beta}$ is the corresponding isotropic autocovariance function. For $d=1$, it will be convenient to view $\beta$ and $\widehat{\beta}$ as even functions on all of $\mathbb{R}$ (e.g., in Section 2) and I will do so without further comment.

The condition (2) has proven useful in distinguishing between models for which a screening effect holds and when it does not. Note that (2) is satisfied by many common models, including all Matérn models (Stein, 1999), all rational spectral densities and a class of spectral densities proposed in Stein (2005, equation (4)) as a model for space-time processes that allows for arbitrary and different degrees of smoothness of the process in space and time. Stein $(2002,2011)$ gives some general results on when a screening effect holds and Stein $(2002,2005,2011)$ gives a number of examples of spectral densities not satisfying (2) that also do not satisfy an asymptotic screening effect as defined by (1). For example, as shown in Section 2, the triangular autocovariance function for a process on $\mathbb{R}$ does not satisfy a screening effect. Its spectral density is of the form $(1-\cos \theta u) /\left(\pi u^{2}\right)$ for some $\theta>0$, which does not satisfy (2) because it "wiggles" at high frequencies. As a second example, consider the squared exponential (sometimes called "Gaussian") autocovariance function $e^{-(r / \theta)^{2}}$, which, for a process on $\mathbb{R}$, has corresponding spectral density $(\theta / \sqrt{4 \pi}) e^{-\theta^{2} \omega^{2} / 4}$. This spectral density does not satisfy (2) because it decreases too quickly at high frequencies. To show that this model does not satisfy a screening effect, consider $N_{\epsilon}=\left\{\epsilon, 2 \epsilon, \ldots, n_{\epsilon} \epsilon\right\}$ and $F_{\epsilon}=\left\{\left(n_{\epsilon}+1\right) \epsilon\right\}$. Under this model, for evenly spaced observations with spacing $\epsilon$, the $j$ 'th diagonal element of the Cholesky decomposition of the covariance matrix of the observation equals $\prod_{\ell=1}^{j-1}\left(1-e^{-2 \ell(\epsilon / \theta)^{2}}\right)^{1 / 2}$ (Lam and Loh, 2000). Because the $j$ 'th diagonal element of the Cholesky decomposition is the standard deviation of the error of the optimal predictor of the $j$ 'th observation given the previous $j-1$ observations,

$$
\frac{E e\left(N_{\epsilon} \cup F_{\epsilon}\right)^{2}}{E e\left(N_{\epsilon}\right)^{2}}=1-\exp \left\{-2 n_{\epsilon}\left(\frac{\epsilon}{\theta}\right)^{2}\right\} \text {. }
$$

If, for example, $\epsilon=1 / n$ and $n_{\epsilon}=n$, then this ratio is $1-e^{-2 /\left(n \theta^{2}\right)}$, which 
tends to 0 as $n \rightarrow \infty$, so even though $F_{\epsilon}$ consists of only 1 observation and $N_{\epsilon}$ consists of $n$, the screening effect is strongly violated. A third example is given by separable models, in which both the spectral density and the corresponding autocovariance function factor into functions of each coordinate. For example, if $\omega=\left(\omega_{1}, \omega_{2}\right) \in \mathbb{R}^{2}$ and $f(\omega)=1 /\left\{\left(1+\omega_{1}^{2}\right)\left(1+\omega_{2}^{2}\right)\right\}$, then (2) is not satisfied, which can be seen by taking $\omega=\left(\omega_{1}, 0\right)$ and $\nu=\left(0, \omega_{2}\right)$ with $\omega_{2} \neq 0$ fixed and letting $\omega_{1} \rightarrow \infty$. For this $f$, Stein $(2005,2011)$ shows that one can choose $N_{\epsilon}$ and $F_{\epsilon}$ so that a screening effect does not hold, but I omit the details here because this model is not isotropic.

To summarize previous results, we have that (2) does imply an asymptotic screening effect (1) under various conditions on the observation locations. Furthermore, for a range of examples that look qualitatively quite different but share the common feature that the spectral densities do not satisfy (2), the screening effect does not hold. However, there are no general results showing that spectral densities not satisfying (2) generally violate the screening effect for some "reasonable" choices of $N_{\epsilon}$ and $F_{\epsilon}$.

Let us first review some mathematical notation that will be used throughout this work. Write $f(x) \sim g(x)$ as $x \rightarrow x_{0}$ ( $x_{0}$ may be infinite) if $\lim _{x \rightarrow x_{0}} f(x) / g(x)=1$ and, for a positive function $h$, write $f(x)=g(x)+$ $o(h(x))$ as $x \rightarrow x_{0}$ if $\lim _{x \rightarrow x_{0}}|f(x)-g(x)| / h(x)=0$. For two positive functions $f$ and $g$ on a domain $D$, write $f \asymp g$ if there exists $0<C_{1}<C_{2}<\infty$ such that $C_{1}<f(x) / g(x)<C_{2}$ for all $x \in D$. Say that $f \asymp g$ as $x \rightarrow x_{0}$ if $f \asymp g$ in some neighborhood of $x_{0}$. For real $x$, denote by $\lfloor x\rfloor$ the greatest integer less than or equal to $x$.

To describe the results of this paper, it is helpful to delineate the relationship between the high frequency behavior of an isotropic spectral density $f$ on $\mathbb{R}^{d}$ and the behavior of its Fourier transform in a neighborhood of the origin. Define the tail area for $f$ by $T_{f}(u)=A_{d} \int_{u}^{\infty} r^{d-1} f(r) d r$, where $A_{d}$ is the surface of the unit sphere in $d$ dimensions. Suppose, for some positive $\alpha$ and $C$,

$$
T_{f}(u) \sim \frac{C}{u^{\alpha}}
$$


as $u \rightarrow \infty$ and let $p=\lfloor\alpha / 2\rfloor$. Then by a Tauberian theorem for Hankel transforms (see, e.g., Bingham, 1972, Theorem 2),

$$
\widehat{f}(r)=\sum_{j=0}^{p} \rho_{j} r^{2 j}+C B_{\alpha, d}\langle r\rangle^{\alpha}+o\left(\langle r\rangle^{\alpha}\right)
$$

as $r \downarrow 0$, where $\langle r\rangle^{\alpha}=r^{\alpha}$ if $\alpha$ is not an even integer and $\langle r\rangle^{\alpha}=r^{\alpha} \log r$ if it is (see Bingham (1972) for an explicit expression for $B_{\alpha, d}$ ). The term $C B_{\alpha, d}\langle r\rangle^{\alpha}$ is known as the principal irregular term of $\widehat{f}$ and it controls the local behavior of a random field $Z$ with mean 0 and this autocovariance function. In particular, $Z$ has $p$ mean square derivatives in any direction if and only if $\alpha / 2>p$. Natural processes in the environment often appear not to be all that smooth, so that in these applications $\alpha$ is generally less than 6 (so fewer than 3 times differentiable), but for modeling the output of computer experiments as a function of some parameters of the model, very large values of $\alpha$ may be appropriate.

Section 2 considers two instructive examples for spectral densities that demonstrate the difficulties in obtaining conditions under which (1) does not hold. The first example is the triangular model, a valid model on $\mathbb{R}$ with spectral density

$$
\kappa_{\theta}(u)=\frac{1-\cos \theta u}{\pi u^{2}}
$$

for $\theta>0$ and for which $\widehat{\kappa}_{\theta}(x)=(\theta-|x|)^{+}$(the superscript plus indicates positive part), so that $-|x|$ is the principal irregular term. Note that $\widehat{\kappa}_{\theta}(x)$ is not differentiable at $\pm \theta$ and that $\theta$ is the coefficient multiplying $u$ in the cosine term in (5). Proposition 1 in Section 2 shows this connection between a periodicity in a spectral density $f$ on $\mathbb{R}$ and a lack of smoothness of $\widehat{f}$ at a specific lag holds more generally. The triangular model does not satisfy (2) and (see Section 2, Stein and Handcock, 1989 and Stein, 1999) it also does not satisfy the screening effect (1) for various choices of $N_{\epsilon}$ and $F_{\epsilon}$. The second example in Section 2 is a spectral density on $\mathbb{R}$ with oscillating term whose period of oscillation tends to 0 as frequency increases:

$$
h(u)=\frac{\sin \left(u^{2} /(2 \pi)\right)}{\pi u^{2}}+\frac{\psi_{0}+u^{2}}{\pi\left(1+u^{2}\right)^{2}},
$$


where $\psi_{0}=2+1 /\left(2 \pi^{2}\right)$ makes $h$ nonnegative, which can be proven by noting that $h$ is trivially nonnegative for $u^{2} /(2 \pi) \leq \pi$ and by using the lower bound $\sin \left(u^{2} /(2 \pi)\right) \geq-1$ and some simple algebra when $u^{2} /(2 \pi)>\pi$. Like the triangular model, from (10) below, it follows that $\widehat{h}(x)$ also has $-|x|$ as its principal irregular term. In contrast to the triangular case, though, $\widehat{h}$ possesses derivatives of all orders for all $x \neq 0$ despite the rapid oscillations at high frequencies in $h$ (see Section 2). Furthermore, again in contrast with the triangular model, under some natural constraints on $N_{\epsilon}$, whenever $U_{\epsilon}$ is contained in a common bounded interval for all $\epsilon$, this model does satisfy (1) (see Section 2).

Section 3 considers a prediction problem that identifies a broad range of spectral densities for which the behavior of the autocovariance function at the origin does not determine the limiting behavior of the optimal predictor. The formulation supposes the isotropic process $Z$ is observed on all of $\mathbb{R}^{d}$ with white noise and looks at the limiting behavior of the optimal linear predictor of $Z(0)$ as the variance of the white noise tends to 0 . Specifically, consider two isotropic models whose tail areas both satisfy (3), so that their corresponding Fourier transforms have the same principal irregular term. Proposition 2 in Section 3 shows that if one of the spectral densities is "wiggly" at high frequencies and the other is not, the optimal linear predictors under the wiggly model are asymptotically nonnegligibly better than under the well-behaved spectral density. In addition, for wiggly spectral densities of a more restrictive form, Proposition 3 gives an explicit asymptotic form for the relative advantage in mean squared error for the wiggly spectral density relative to the well-behaved alternatives as the white noise variance tends to 0 . Both of these results cover the spectral density $h$ in (6) and show that predictions under this model are nontrivially better than under an exponential model, despite the fact that both corresponding autocovariance functions have principal irregular term $-|x|$.

These results are of a rather different form than of an asymptotic screening effect as defined in (1). At least in some special cases that include the two 
examples from Section 2, Section 3.1 shows that a result of the form (1) fails to hold for this white noise formulation. There is also some brief discussion on the role of white noise models in statistics to obtain asymptotic results and how they can be used to obtain results in other asymptotic regimes. Section 4 describes numerical results for a specific prediction problem under $h$ and discusses their relevance to a common approach to likelihood approximation. Section 5 discusses implications of the findings in the previous sections for modeling of spatial processes and topics for further research.

\section{Two instructive examples}

Spectral densities not satisfying (2) often correspond to autocovariance functions that have some lack of smoothness away from the origin. Proposition 1 shows that the relationship we saw for the triangular model between oscillations at high frequencies of a spectral density on $\mathbb{R}$ and lack of smoothness away from the origin of its Fourier transform holds more generally. A function $f$ on $\mathbb{R}$ is said to be infinitely differentiable at some point $x \in \mathbb{R}$ if $f^{(m)}(x)$ exists for all positive integers $m$.

Proposition 1. Consider a spectral density $f$ on $\mathbb{R}$ of the form

$$
f(u)=(a+b \cos \theta u) q(u),
$$

where $a>|b|>0$ and, for some $\alpha>0, q$ is even and satisfies

$$
q^{(m)}(u) \asymp(1+|u|)^{-m-\alpha-1}
$$

for all nonnegative integers $m$. Then $\widehat{f}$ is infinitely differentiable for all $x \in \mathbb{R}$ except for $x=0, \theta$ and $-\theta$ and it has the same number of derivatives at these three $x$ values.

The condition (8) is satisfied if, for example, $q(u)=\phi\left(\beta^{2}+u^{2}\right)^{-(\alpha+1) / 2}$ with $\phi, \alpha$ and $\beta$ positive, which is the form for the spectral density of a Matérn model on $\mathbb{R}$ (Stein, 1999, p. 31). To prove this proposition, write

$$
\int_{-\infty}^{\infty} q(u) \cos \theta u \cos r u d u=\frac{1}{2} \int_{-\infty}^{\infty} q(u)[\cos \{(\theta-r) u\}+\cos \{(\theta+r) u\}] d u .
$$


Using (8), Proposition 3 in Stein (2005) implies that $\widehat{q}(x)=\int_{-\infty}^{\infty} q(u) \cos x u d u$ is infinitely differentiable in $x$ for $x \neq 0$. Examining (7) and (9), we see that $\widehat{f}$ is infinitely differentiable for $x \neq 0, \pm \theta$ and has the same number of derivatives at $0, \theta$ and $-\theta$, completing the proof of the proposition.

Considering (4), one might expect $\widehat{q}^{(2 k)}(0)$ exists if and only if $\alpha>2 k$, which is indeed the case (Stein, 1999, p. 27). Thus, $\widehat{f}$ has $2 k$ derivatives at $\pm \theta$ if and only if it has $2 k$ derivatives at 0 . It is possible to make more specific statements about the relationship between $\widehat{f}$ at 0 and at $\pm \theta$. If, for example, $q(u) \sim C u^{-\alpha-1}$ as $u \rightarrow \infty$ and $\alpha=2 p$ for nonnegative integer $p$, then (Stein, 1999, p. 34) $\widehat{f}^{(2 p+1)}\left(0^{+}\right)-\widehat{f}^{(2 p+1)}\left(0^{-}\right)=a C^{\prime}$ with $C^{\prime}=2(-1)^{p+1} \pi C /(2 p+1)$ ! and $\widehat{f}^{(2 p+1)}\left(\theta^{+}\right)-\widehat{f}^{(2 p+1)}\left(\theta^{-}\right)=b C^{\prime} / 2$. For the triangular autocovariance function, $a=1$ and $b=-1$ and, correspondingly, $\widehat{\kappa}_{\theta}^{\prime}\left(0^{+}\right)-\widehat{\kappa}_{\theta}^{\prime}\left(0^{-}\right)=-2$ and $\widehat{\kappa}_{\theta}^{\prime}\left(\theta^{+}\right)-\widehat{\kappa}_{\theta}^{\prime}\left(\theta^{-}\right)=1$. Note that $\kappa_{\theta}(u)=(1-\cos \theta u) /\left(\pi u^{2}\right)$ does not quite satisfy (8) because $q(u)=u^{-2}$ is unbounded in a neighborhood of 0 (even though $f$ is bounded), but the differentiability of Fourier transforms is unaffected by the behavior of the spectral density on bounded intervals, so the conclusions about the lack of smoothness of $\widehat{\kappa}_{\theta}$ at $\theta$ still apply.

This lack of smoothness away from the origin of $\widehat{\kappa}_{\theta}$ is in turn closely related to the lack of a screening effect for this model. For this model, for $0<\epsilon<\theta$, consider predictions of $Z(0)$ when $N_{\epsilon}=\{\epsilon\}$ and $F_{\epsilon}=\{\theta, \theta+\epsilon\}$. The optimal predictor of $Z(0)$ based on the observation in $N_{\epsilon}$ is $(1-\epsilon / \theta) Z(\epsilon)$ with mean squared error $2 \epsilon-\epsilon^{2} / \theta$; based on observations in $N_{\epsilon} \cup F_{\epsilon}$, direct calculation shows the optimal predictor is

$$
\left(1-\frac{\epsilon}{2 \theta}\right) Z(\epsilon)-\frac{1}{2} Z(\theta)+\left(\frac{1}{2}-\frac{\epsilon}{2 \theta}\right) Z(\theta+\epsilon)
$$

with mean squared error $(3 / 2) \epsilon-\epsilon^{2} /(2 \theta)$. The limit in (1) is thus $3 / 4$ rather than 1. This limit is unchanged if $N_{\epsilon}$ is the interval $[\epsilon, \theta]$.

This example suggests a close connection between a condition like (2) on $f$, the smoothness of $\widehat{f}$ away from the origin, and the presence of an asymptotic screening effect. Stein (2005) gives further results on the relationship between $f$ and the smoothness of $\widehat{f}$ away from the origin. However, there 
are no general inverses to these results when $f$ does not satisfy a condition like (2). Although Proposition 1 here does give a limited result on the lack of differentiability of $\widehat{f}$ away from the origin when $f$ does not satisfy (2), the spectral density $h$ in (6) illustrates the difficulties in obtaining a more general inverse. The first term in (6) oscillates rapidly at high frequencies, so we might expect $\widehat{h}$ to have some lack of smoothness away from the origin. However, the frequency of oscillation continually changes with $u$ so that we cannot match this oscillation over a broad range of frequencies with $\cos x u$ for any given $x$. It turns out that $\widehat{h}$ is infinitely differentiable for $x \neq 0$ and is given by

$$
\begin{aligned}
\widehat{h}(x)= & x\{S(x)-C(x)\}+\frac{\sqrt{2}}{\pi} \sin \left(\frac{\pi x^{2}}{2}+\frac{\pi}{4}\right) \\
& +\frac{1}{2}\left\{\psi_{0}+1+\left(\psi_{0}-1\right)|x|\right\} e^{-|x|},
\end{aligned}
$$

where $S$ and $C$ are the Fresnel integrals (Olver, et al., 2010, Ch. 7)

$$
S(x)=\int_{0}^{x} \sin \left(\frac{1}{2} \pi t^{2}\right) d t
$$

and

$$
C(x)=\int_{0}^{x} \cos \left(\frac{1}{2} \pi t^{2}\right) d t .
$$

This result follows from 1.7(3) in Erdelyi (1954), although the definitions of $S$ and $C$ used on p. 387 of Erdelyi (1954) are not the appropriate ones for (10) to hold. Straightforward calculus shows that for $x \neq 0$,

$$
\widehat{h}^{(2)}(x)=-\sqrt{2} \cos \left(\frac{\pi x^{2}}{2}+\frac{\pi}{4}\right)+\frac{1}{2}\left\{\psi_{0}+1-\left(\psi_{0}-1\right)|x|\right\} e^{-|x|}
$$

and it follows that $\widehat{h}^{(2)}(x)$ does not tend to 0 as $x \rightarrow \infty$ and the third and higher derivatives of $\widehat{h}$ are unbounded as $x \rightarrow \infty$. Thus, there is a sense in which $\widehat{h}(x)$ is "not smooth" away from the origin even though it is infinitely differentiable for $x \neq 0$.

Next, let us consider whether a screening effect holds for $\widehat{h}$. Since $x S(x)$ and $x C(x)$ are both analytic in $x$, the lack of differentiability in $\widehat{h}$ is controlled 
by the last term on the right-hand side of (10). It then follows that for any positive and finite $T$, the probability measure for this Gaussian process on the interval $[0, T]$ with mean 0 and autocovariance function $\widehat{h}$ is equivalent to the measure for the Gaussian process with mean 0 and exponential autocovariance function $K_{0}(x)=e^{-|x|}$ (Ibragimov and Rozanov, 1978, Theorem 13 , Ch. 3), since $\int_{0}^{T} \int_{0}^{T}\left\{K_{0}^{(2)}(x-y)-\widehat{h}^{(2)}(x-y)\right\}^{2} d x d y<\infty$. We can use this equivalence of measures to show that screening effects will hold for $\widehat{h}(x)$ when observations are restricted to a bounded interval. For example, suppose $x_{1}, x_{2}, \ldots$ is a sequence of nonzero points in some bounded interval including 0 as a limit point. Let $U_{\epsilon}=\left\{x_{1}, \ldots, x_{n_{\epsilon}}\right\}$, where $n_{\epsilon}=\lfloor 1 / \epsilon\rfloor$ and $N_{\epsilon}$ is made up of the smallest positive and largest negative points in $U_{\epsilon}$ (so containing at most two observations). Label $\widehat{h}=K_{1}$ and write, for $j=0,1, e_{j}(S)$ for the optimal linear predictor of $Z(0)$ under autocovariance function $K_{j}$ based on observations in the set $S$ and $E_{j}$ for expectations under $K_{j}$. Because the process is exactly Markov under $K_{0}$, we have $E_{0} e_{0}\left(N_{\epsilon}\right)^{2}=E_{0} e_{0}\left(U_{\epsilon}\right)^{2}$. Since the Gaussian measures on this observation interval defined by $K_{0}$ and $K_{1}$ are equivalent, by Theorem 10 in Ch. 4 of Stein (1999), $E_{0} e_{0}\left(U_{\epsilon}\right)^{2} \sim E_{0} e_{1}\left(U_{\epsilon}\right)^{2} \sim E_{1} e_{0}\left(U_{\epsilon}\right)^{2} \sim E_{1} e_{1}\left(U_{\epsilon}\right)^{2}$ as $\epsilon \downarrow 0$. Direct calculation shows $E_{0} e_{0}\left(N_{\epsilon}\right)^{2} \sim E_{1} e_{1}\left(N_{\epsilon}\right)^{2}$ as $\epsilon \downarrow 0$. Thus, (1) holds for $\widehat{h}=K_{1}$ and this definition of $N_{\epsilon}$ and $F_{\epsilon}$.

This equivalence of measures on any bounded interval implies that to show $\widehat{h}$ does not satisfy a screening effect, it is necessary to consider observations not constrained to a bounded interval. We could consider an infinite lattice of observations as in Stein (2002), and I conjecture that a screening effect will not hold in this case, but the calculations are rather messy as one needs to consider infinite sums of $h$ over a lattice of frequencies.

\section{Main Results}

This section shows how supposing the isotropic process $Z$ is observed with white noise on all of $\mathbb{R}^{d}$ leads to tractable calculations of asymptotic mean squared prediction error even for spectral densities like $h$. Let $W$ be white 
noise, the generalized random field (Yaglom, 1987, Sec. 24 and 25) with constant spectral density $\epsilon$, for which $\operatorname{var}\left(\int v(x) W(x) d x\right)=\epsilon(2 \pi)^{d} \int v(x)^{2} d x$ for all square integrable $v$. The use of the same symbol $\epsilon$ for a quantity tending to 0 in Section 2 and here is deliberate and reflects the notion that for $\epsilon$ small, in both settings, the observations provide a lot of information about how $Z$ behaves in a neighborhood of the origin. (More specifically, as $\epsilon \downarrow 0$, $Z(0)$ and any existing mean square derivatives of $Z$ at 0 can be predicted with mean squared error tending to 0 ; see Stein (2011) for further discussion.) Suppose $Z$ and $W$ are independent and define $Y(x)=Z(x)+W(x)$. Consider predicting $Z(0)$ based on a predictor of the form $\int v(x) Y(x) d x$ for $v$ square integrable. The mean squared prediction error for the optimal $v$ then takes on the particularly simple form

$$
V_{\epsilon}(f)=A_{d} \epsilon \int_{0}^{\infty} \frac{f(u) u^{d-1}}{\epsilon+f(u)} d u
$$

which follows from standard results on the non-causal Wiener filter (Wiener, 1949). Note that this result is unchanged if $Z$ is assumed to have an unknown constant mean since this mean can be (almost surely) estimated without error based on $Y$.

Suppose $f$ satisfies (3) for some positive $C$ and $\alpha$. Define $\bar{C}=\alpha C / A_{d}$ and $g(u)=\bar{C} u^{-\alpha-d}$, so that $T_{g}(u)=A_{d} \int_{u}^{\infty} g(r) r^{d-1} d r=C u^{-\alpha}$. Note that $g$ is not a spectral density in the ordinary sense, since it is not integrable in a neighborhood of the origin. However, similar to (4), it is the spectral density for an intrinsic random function of order $p=\lfloor\alpha / 2\rfloor$ (Chilès and Delfiner, 2012) whose Fourier transform (properly defined) is the generalized autocovariance function $C B_{\alpha, d}\langle r\rangle^{\alpha}$, which describes the variances of all increments of order $p$ of $Z$. Furthermore, as long as the optimal predictor is defined appropriately (only predictors whose error is an increment of order $p$ are allowed), (11) still applies, and a simple change of variables shows (using 
3.241(4) in Gradshteyn and Ryzhik (2007))

$$
\begin{aligned}
V_{\epsilon}(g) & =\epsilon^{\alpha /(\alpha+d)} A_{d} \bar{C}^{d /(\alpha+d)} \int_{0}^{\infty} \frac{r^{d-1}}{1+^{\alpha+d}} d r \\
& =\epsilon^{\alpha /(\alpha+d)} \bar{C}^{d /(\alpha+d)} \frac{A_{d}}{\alpha+d} B\left(\frac{d}{\alpha+d}, \frac{\alpha}{\alpha+d}\right),
\end{aligned}
$$

where $B(\cdot, \cdot)$ is the beta function. Suppose $f(u) \sim g(u)$ as $u \rightarrow \infty$. Writing $\tau(\epsilon)=\epsilon^{-1 /\{2(\alpha+d)\}}$,

$$
V_{\epsilon}(f)=A_{d} \epsilon \int_{0}^{\tau(\epsilon)} \frac{f(u) u^{d-1}}{\epsilon+f(u)} d u+A_{d} \epsilon \int_{\tau(\epsilon)}^{\infty} \frac{f(u) u^{d-1}}{\epsilon+f(u)} d u .
$$

It is then possible to show that the first term on the right-hand side of this equation is $o\left(\epsilon^{\alpha /(\alpha+d)}\right)$ as $\epsilon \downarrow 0$ and

$$
\int_{\tau(\epsilon)}^{\infty} \frac{f(u) u^{d-1}}{\epsilon+f(u)} d u \sim \int_{\tau(\epsilon)}^{\infty} \frac{g(u) u^{d-1}}{\epsilon+g(u)} d u \sim \int_{0}^{\infty} \frac{g(u) u^{d-1}}{\epsilon+g(u)} d u
$$

as $\epsilon \downarrow 0$. Thus, $f(u) \sim g(u)$ as $u \rightarrow \infty$ implies $V_{\epsilon}(f) \sim V_{\epsilon}(g)$ as $\epsilon \downarrow 0$.

Now let us consider $f$ satisfying $(3)$ but $f(u) \sim g(u)$ as $u \rightarrow \infty$ does not hold. In particular, suppose

$$
\limsup _{u \rightarrow \infty} \frac{f(u)}{g(u)}<\infty
$$

and

$$
\int_{u}^{\infty}\{f(r)-g(r)\}^{2} r^{d-1} d r \asymp u^{-2 \alpha-d}
$$

as $u \rightarrow \infty$. Since $\int_{u}^{\infty} g(r)^{2} r^{d-1} d r \asymp u^{-2 \alpha-d}$, (14) implies that $f$ fluctuates nontrivially at high frequencies, despite the fact that both $f$ and $g$ have corresponding (generalized) autocovariance functions with principal irregular term $C B_{\alpha, d}\langle r\rangle^{\alpha}$. The following result, proven in the Appendix, indicates that this principal irregular term does not determine the asymptotic mean squared prediction error for $Z(0)$ as $\epsilon \downarrow 0$ under $f$.

Proposition 2. Suppose $f$ satisfies (3), (13) and (14) and $g(u)=\bar{C} u^{-\alpha-d}$. Then

$$
\limsup _{\epsilon \downarrow 0} \frac{V_{\epsilon}(f)}{V_{\epsilon}(g)}<1 \text {. }
$$


Under stronger conditions on $f$, Proposition 3 shows how much better, asymptotically, $Z(0)$ can be predicted based on $f$ rather than $g$.

Proposition 3. Suppose $f$ satisfies (3),

$$
\lim _{u \rightarrow \infty} f(u) u^{\alpha+d}-P(\rho(u))=0,
$$

where $P$ is a bounded, measurable, nonnegative function on $[0, \infty)$ with period $1, \bar{C}=\int_{0}^{1} P(t) d t>0, \rho(0)=0, \rho$ has a positive derivative on $[0, \infty)$,

$$
\lim _{u \rightarrow \infty} u \rho^{\prime}(u)=\infty
$$

and for any two sequences $\left\{s_{n}\right\}$ and $\left\{t_{n}\right\}$ of positive numbers satisfying $s_{n} \rightarrow$ $\infty$ and $\left(s_{n}-t_{n}\right) / s_{n} \rightarrow 0$ as $n \rightarrow \infty$,

$$
\lim _{n \rightarrow \infty} \frac{\rho^{\prime}\left(s_{n}\right)-\rho^{\prime}\left(t_{n}\right)}{\rho^{\prime}\left(s_{n}\right)}=0 .
$$

Then as $\epsilon \downarrow 0$,

$$
\frac{V_{\epsilon}(f)}{V_{\epsilon}(g)} \sim \bar{C}^{-d /(\alpha+d)} \int_{0}^{1} P(t)^{d /(\alpha+d)} d t
$$

where $g(u)=\bar{C} u^{-\alpha-d}$.

See the Appendix for a proof. The conditions (16) and (17) ensure that $\rho(u)$ grows sufficiently quickly as $u \rightarrow \infty$ and that $\rho^{\prime}(u)$ changes sufficiently slowly for $u$ large. For example, all functions of the form $u^{\gamma} \log ^{\beta}(1+u)$ satisfy all the conditions on $\rho$ when $\gamma>0$ and $\beta \geq 0$, but (16) is not satisfied when $\gamma=0$ for any $\beta$. Because $0<d /(\alpha+d)<1, \int_{0}^{1} P(t)^{d /(\alpha+d)} d t \leq \bar{C}^{d /(\alpha+d)}$ with equality if and only if $P(t)=\bar{C}$ almost everywhere on $[0,1]$. Note that the asymptotic mean squared error does not depend on the specific form of $\rho$ as long as it satisfies the conditions in the proposition. For the triangular spectral density $\kappa_{\theta}$ in $(5), P(t)$ can be taken as $(1-\cos 2 \pi t) / \pi$ (independent of $\theta$ ) and $\rho(u)=\theta u /(2 \pi)$. For the spectral density $h$ in (6), $P(t)$ can be taken as $(1+\sin 2 \pi t) / \pi$ and $\rho(u)=(u /(2 \pi))^{2}$. Since $\alpha=1$ for both of these spectral densities, it follows that the limit on the right hand side of (18) equals $\int_{0}^{1} \sqrt{1-\cos 2 \pi t} d t$ in both cases. Simple calculus shows this integral 
equals $2 \sqrt{2} / \pi \approx .9003$. To give one more example, consider the commonly used spherical autocovariance function, which, for some $\theta>0$, equals $1-$ $1.5(r / \theta)+0.5(r / \theta)^{3}$ for interpoint distances $r$ less than $\theta$ and is 0 otherwise. This model is a valid isotropic covariance in up to $d=3$ dimensions and, for $d=3$, its spectral density is of the form $f(u)=3 \pi u^{-4} \cos ^{2}(\theta u)+O\left(u^{-5}\right)$ as $u \rightarrow \infty$ and thus satisfies the conditions of Proposition 3 with $\alpha=1$ and $P(t)=1.5 \pi(1+\cos 2 \pi t)$. The right hand side of (18) thus equals $\int_{0}^{1}(1+$ $\cos 2 \pi t)^{3 / 4} d t=2^{1 / 4} \Gamma(1 / 4)^{2} /\left(3 \pi^{3 / 2}\right) \approx .9358$ by 3.621(3) in Gradshteyn and Ryzhik (2007). See Stein and Handcock (1989) for another example of how the spherical model does not satisfy a screening effect.

\subsection{Relation to screening effect}

Proposition 2 does not specifically show that something like (1) does not hold under the conditions of the proposition. This section shows that for some $f$ and $N_{\epsilon}$ and $F_{\epsilon}$ defined appropriately, (1) is false when $Z$ is observed with white noise. Define $V_{\epsilon, B}(f)$ to be the mean squared error of the optimal predictor of $Z(0)$ based on observing $Z$ plus white noise with variance $\epsilon$ over the domain $B$. Write $V_{\epsilon, r}$ if $B$ is the ball of radius $r$ centered at 0 , so that $V_{\epsilon, \infty}(f)=V_{\epsilon}(f)$. Using a substantially simplified version of the proof of Theorem 1 in Stein (2002), it is possible to prove that if $f(u) \sim g(u)=$ $\bar{C} u^{-\alpha-d}$ as $u \rightarrow \infty$ for some positive $\bar{C}$ and $\alpha$, then

$$
\lim _{\epsilon \downarrow 0} \frac{V_{\epsilon}(g)}{V_{\epsilon, r_{\epsilon}}(f)}=\lim _{\epsilon \downarrow 0} \frac{V_{\epsilon}(f)}{V_{\epsilon, r_{\epsilon}}(f)}=1
$$

whenever $r_{\epsilon}^{d-1} / \epsilon \rightarrow \infty$ as $\epsilon \downarrow 0$. This condition on $r_{\epsilon}$ allows for $Z(0)$ to be predicted with mean squared error tending to 0 as $\epsilon \downarrow 0$. I would conjecture that, under the conditions on $f$ in Proposition 2 and if $r_{\epsilon}^{d-1} / \epsilon \rightarrow \infty$ but $r_{\epsilon} \rightarrow 0$ as $\epsilon \downarrow 0$, then

$$
\limsup _{\epsilon \downarrow 0} \frac{V_{\epsilon}(f)}{V_{\epsilon, r_{\epsilon}}(f)}<1 .
$$

This result would follow from Proposition 2 if, under the same restrictions on $r_{\epsilon}$,

$$
\lim _{\epsilon \downarrow 0} \frac{V_{\epsilon, r_{\epsilon}}(g)}{V_{\epsilon, r_{\epsilon}}(f)}=1
$$


which may appear plausible given that $f$ and $g$ corresponding to (generalized) autocovariance functions with the same principal irregular term, but I do not have a proof.

For certain special cases, it is possible to prove (20). In particular, if spectral densities $f_{0}$ and $f_{1}$ correspond to equivalent Gaussian measures on some domain $B$ with 0 in its interior, then, similar to the proof of Theorem 10 in Chapter 4 of Stein (1999), it is possible to show that

$$
\lim _{\epsilon \downarrow 0} \frac{V_{\epsilon, B}\left(f_{0}\right)}{V_{\epsilon, B}\left(f_{1}\right)}=1 .
$$

Consider the two examples from Section 2: the triangular model with spectral density $\kappa_{\theta}$ as in (5) and $h$ as given in (6). Letting $f_{0}(u)=1 /\left\{\pi\left(1+u^{2}\right)\right\}$, then on any interval of length at most $\theta$, the Gaussian measures corresponding to $f_{0}$ and $\kappa_{\theta}$ are equivalent, and for any finite interval, the Gaussian measures corresponding to $f_{0}$ and $h$ are equivalent. From (22),

$$
\lim _{\epsilon \downarrow 0} \frac{V_{\epsilon, r}\left(f_{0}\right)}{V_{\epsilon, r}\left(\kappa_{\theta}\right)}=1
$$

for $r \leq \theta / 2$ and

$$
\lim _{\epsilon \downarrow 0} \frac{V_{\epsilon, r}\left(f_{0}\right)}{V_{\epsilon, r}(h)}=1
$$

for all $r<\infty$. Consider

$$
\frac{V_{\epsilon, r}\left(\kappa_{\theta}\right)}{V_{\epsilon}\left(\kappa_{\theta}\right)}=\frac{V_{\epsilon, r}\left(\kappa_{\theta}\right)}{V_{\epsilon, r}\left(f_{0}\right)} \times \frac{V_{\epsilon, r}\left(f_{0}\right)}{V_{\epsilon}\left(f_{0}\right)} \times \frac{V_{\epsilon}\left(f_{0}\right)}{V_{\epsilon}\left(\kappa_{\theta}\right)} .
$$

For $r \leq \theta / 2$, as $\epsilon \downarrow 0$, the first term on the right-hand side of (25) tends to 1 by (23), the second term tends to 1 by (19), and the third term has a lim sup less than 1 by Proposition 2. Thus, for all $r \leq \theta / 2$,

$$
\limsup _{\epsilon \downarrow 0} \frac{V_{\epsilon, r}\left(\kappa_{\theta}\right)}{V_{\epsilon}\left(\kappa_{\theta}\right)}<1 .
$$

Similarly, because of (24), for all $r$ finite,

$$
\limsup _{\epsilon \downarrow 0} \frac{V_{\epsilon, r}(h)}{V_{\epsilon}(h)}<1 .
$$


These results are even stronger than (20), which assumed $r_{\epsilon} \rightarrow 0$ as $\epsilon \downarrow 0$.

The use of white noise models as a way of simplifying the mathematics for obtaining asymptotic results in statistical inference goes back at least to Pinsker (1980). In the context of nonparametric regression, Brown and Low (1996) developed an equivalence theory (using a notion of equivalence based on Lecam's distance between statistical experiments, not the notion of equivalence of Gaussian measures in Section 2) showing that results obtained under white noise models could be used to obtain parallel results in the more practical setting of a function observed with independent and identically distributed errors on an increasingly dense grid. This approach has been succesfully applied to a number of other problems in statistics (see, e.g., Brown, Carter, Low and Zhang, 2004 and Golubev, Nussbaum and Zhou, 2010) and it seems plausible that it could be applied to prove versions of Propositions 2 and 3 when $Z$ is observed at $Z(\epsilon j)$ with independent and identically distributed errors at all $j \in \mathbb{Z}^{d}$.

\section{Numerical results}

Observing $Z$ everywhere on $\mathbb{R}^{d}$ with white noise simplifies the mathematical challenges of studying the screening effect, but is not of the most practical relevance. A more relevant formulation is to consider predicting $Z(0)$ based on observations $Z(\epsilon j)$ for $j=1, \ldots, J$. This is both a more interesting prediction problem and it is directly relevant to an important method of likelihood approximation for Gaussian processes. Specifically, Vecchia (1988) proposed approximating the likelihood of a set of spatial observations by ordering the observations in some manner, say as $z_{1}, \ldots, z_{n}$, and then approximating the likelihood by $p_{\theta}\left(z_{1}\right) \prod_{j=2}^{n} p_{\theta}\left(z_{j} \mid s_{j}\right)$, where $s_{j}$ is some subset of the "past" observations $\left\{z_{1}, \ldots, z_{j-1}\right\}$ and $p_{\theta}$ is used to indicate the obvious marginal or conditional densities as functions of some unknown parameter vector $\theta$. If $s_{j}=\left\{z_{1}, \ldots, z_{j-1}\right\}$ for $j=2, \ldots, n$, then this approach is the exact likelihood, so if a screening effect holds to a good approximation, we might expect this approximation to work well when $s_{j}$ is taken to be those observations 
among $\left\{z_{1}, \ldots, z_{j-1}\right\}$ that are closest to $z_{j}$. Note that it is not necessarily the case that $s_{j}$ should be chosen to be the closest past observations to $z_{j}$, an issue that has been explored in the prediction context (Rivoirard, 1987, Haslett, 1989, Emery, 2009 and Abedini, et al., 2012) and for likelihood approximation (Stein, et al., 2004). This basic idea has also been used for approximate simulation of Gaussian processes (Emery and Peláez, 2011) and as part of an MCMC approach to approximate posterior distributions for Gaussian processes (Datta, et al., 2014).

Figures 1 and 2 consider optimal linear prediction of $Z(0)$ under the spectral density $h$ in (6). Defining $S(\epsilon, J)=\{\epsilon j: j=1, \ldots J\}$, the plots give $E e(S(\epsilon, J))^{2} / E e(S(\epsilon, 1))^{2}$ for $\epsilon$ ranging from 0.001 to 0.1 and $J$ going up to (for some $\epsilon$ ) 100,000. The Fresnel integrals were calculated using the programs FresnelS and FresnelC in MATLAB and the mean squared prediction errors using the $\mathrm{R}$ program PacfDL in the $\mathrm{R}$ library FitAR, which uses the Durbin-Levinson algorithm (Shumway and Stoffer, 2011, p. 112).

The horizontal axis in both plots is $L=\epsilon J$, so that $L$ is the distance from 0 to the most distant observation. Because of the equivalence of Gaussian measures on all finite intervals between the autocovariance function $\widehat{h}$ and the exponential autocovariance function $e^{-|x|}$, for any fixed $L$,

$$
\lim _{\epsilon \downarrow 0} \frac{E e(S(\epsilon,\lfloor L / \epsilon\rfloor))^{2}}{E e(S(\epsilon, 1))^{2}}=1
$$

(Stein, 1999, Theorem 10, Ch. 4). Figure 1 bears out this result. In contrast, as Figure 2 shows, for sufficiently large $L$, smaller $\epsilon$ values yield smaller ratios of mean squared prediction errors. Thus, we need to let the observations get dense at the same time as the interval of observation lengthens in order to see the lack of an asymptotic screening effect for the spectral density $h$. Setting $J=\infty$ results in the classical Wiener-Kolmogorov prediction problem, for which

$$
E e(S(\epsilon, \infty))^{2}=2 \pi \exp \left[\frac{1}{2 \pi} \int_{-\pi}^{\pi} \log \tilde{h}^{\epsilon}(u) d u\right]
$$


where

$$
\tilde{h}^{\epsilon}(u)=\frac{1}{\epsilon} \sum_{\ell=-\infty}^{\infty} h\left(\frac{u+2 \pi \ell}{\epsilon}\right)
$$

(Stein, 1999, p. 78). Asymptotic analysis of this sum is challenging, but Figure 2 suggests the conjecture

$$
\limsup _{\epsilon \downarrow 0} \frac{E e(S(\epsilon, \infty))^{2}}{E e(S(\epsilon, 1))^{2}}<1
$$

and that perhaps the limit (and not just the limsup) exists and has a value of around 0.73 . Note that if (28) were true, then by (26), we would also have

$$
\limsup _{\epsilon \downarrow 0} \frac{E e(S(\epsilon, \infty))^{2}}{E e(S(\epsilon,\lfloor L / \epsilon\rfloor))^{2}}<1
$$

for any positive $L$. As a consequence, even for very large $J$, the Vecchia approximation may not work all that well for this model when the spacing between observations is sufficiently small and the length of the observation interval sufficiently large.

\section{Discussion}

The screening effect has long been recognized in the geostatistical community (Journel and Huijbregts, 1978) and it is viewed as a natural consequence of (intrinsically) stationary spatial models, at least in those cases in which a nugget effect (a discontinuity at the origin in the autocovariance function) is not too large. Indeed, in discussing the screening effect, Armstrong (1998, p. 109) writes, "Common sense suggests that the estimates will be almost as precise if only the neighboring data are taken into account." Unfortunately, as this and past work of mine has shown (Stein and Handcock, 1989, Stein, 1999, 2002, 2005 and 2011), the screening effect may not hold in well-defined senses for some models. The counterexamples to a screening effect in these previous works all had spectral densities that violated (2), but they were isolated examples and there was no general theory showing when a screening 
effect would not hold. In addition, excluding models for analytic processes such as the squared exponential, all of these examples had autocovariance functions possessing some lack of differentiability away from the origin. This work provides a first step to obtaining general results on when screening does not hold by considering a white noise formulation that leads to relatively simple calculations. Propositions 2 and 3 show that if an isotropic spectral density whose tail area decays algebraically (3) wiggles too much at high frequencies, a screening effect does not hold. These results apply to the spectral density $h$ in (6), for which the corresponding autocovariance function is infinitely differentiable away from the origin. Thus, these propositions provide evidence that the high frequency characteristics of the spectral density are a better marker for identifying anomolous behavior in optimal linear predictions than lack of differentiability away from the origin in autocovariance functions.

The fact that screening does not always hold should not be interpreted as evidence that it is unimportant. In my view, the more appropriate conclusion is that models for which a screening effect does not hold should be used with caution and, as a consequence, one should generally use spectral densities that are "well-behaved" at high frequencies by, for example, at least satisfying (2). Of course, no one is likely to use the autocovariance function $\widehat{h}$ in an application. However, models that do not possess a screening effect, such as the spherical autocovariance for processes on $\mathbb{R}^{3}$ and the squared exponential in any number of dimensions, are used in practice.

For modeling isotropic processes in $d$ dimensions, the results in this work suggest that a reasonable starting place is to consider models, such as the Matérn, whose spectral density $f$ satisfies $f(u) \sim \bar{C} u^{-\alpha-d}$ as $u \rightarrow \infty$ for some positive constants $\bar{C}$ and $\alpha$. There has been a fair amount of research on estimating $\alpha$ (less on $\bar{C}$ ), but mainly for observations on a regular grid. Chan, et al. (1995) and Wu, et al. (2013) consider estimation in the spectral domain and Jakeman and Jordan (1990), Constantine and Hall (1994), Kent and Wood (1997), Davies and Hall (1999) and Chan and Wood (2000, 2004) 
in the spatial domain. See Gneiting, et al. (2012) for a review of this area. Further results for ungridded observations, which is the norm for many types of spatial data, would be most welcome.

The results here are restricted to isotropic models, which leaves out some important examples that are known not to have a screening effect, such as separable models (Stein, 2005, 2011). Moreover, results on screening effects when the process is observed irregularly are particularly limited (Stein, 2011). Thus, there is scope for considerable more work to show when a screening effect does and does not hold.

\section{Acknowledgements}

This material is based upon work supported by the U.S. Department of Energy Office of Science, Office of Advanced Scientific Computing Research, Applied Mathematics program under Award Number DE-SC-0001234 DESC-0011087. The author thanks two reviewers for their many thoughtful suggestions on how to better present the results of this paper.

\section{Appendix A. Proofs}

Proof of Proposition 2: For a function $h$ on $[0, \infty)$, define $u_{\epsilon}(h)$ to be the infimum over $u$ such that $h(t) \leq \epsilon$. Define $u_{\epsilon}=\max \left(u_{\epsilon}(g), u_{\epsilon}(f)\right)$, so that $g(t) \leq \epsilon$ and $f(t) \leq \epsilon$ for all all $t \geq u(\epsilon)$. By the definition of $g, u_{\epsilon}(g) \asymp \epsilon^{-1 /(\alpha+d)}$ and it follows from (13) that $u_{\epsilon} \asymp \epsilon^{-1 /(\alpha+d)}$ as $\epsilon \downarrow 0$. Consider the function $\phi(x)=x /(\epsilon+x)$ on $[0, \infty)$. This function is concave, so that for any nonnegative $x$ and $x_{0}$,

$$
\phi(x) \leq \phi\left(x_{0}\right)+\left(x-x_{0}\right) \phi^{\prime}\left(x_{0}\right)=\frac{x_{0}}{\epsilon+x_{0}}+\frac{\left(x-x_{0}\right) \epsilon}{\left(\epsilon+x_{0}\right)^{2}} .
$$

In addition,

$$
\phi(x)=\phi\left(x_{0}\right)+\left(x-x_{0}\right) \phi^{\prime}\left(x_{0}\right)+\frac{1}{2}\left(x-x_{0}\right)^{2} \phi^{\prime \prime}(\xi)
$$


for some $\xi$ between $x$ and $x_{0}$. Since $\phi^{\prime \prime}(x)=-2 \epsilon /(\epsilon+x)^{3}$ is increasing in $x$, if both $x$ and $x_{0}$ are at most $\epsilon$, then $\phi^{\prime \prime}(\xi) \leq \phi^{\prime \prime}(\epsilon)$ and

$$
\phi(x) \leq \frac{x_{0}}{\epsilon+x_{0}}+\frac{\left(x-x_{0}\right) \epsilon}{\left(\epsilon+x_{0}\right)^{2}}-\frac{\left(x-x_{0}\right)^{2}}{8 \epsilon^{2}} .
$$

From (A.1) and (A.2) and the definition of $u_{\epsilon}$,

$$
\begin{aligned}
V_{\epsilon}(f) \leq & A_{d} \epsilon \int_{0}^{\infty}\left[\frac{g(u)}{\epsilon+g(u)}+\epsilon \frac{f(u)-g(u)}{\{\epsilon+g(u)\}^{2}}\right] u^{d-1} d u \\
& -A_{d} \epsilon^{2} \int_{u_{\epsilon}}^{\infty} \frac{\{f(u)-g(u)\}^{2}}{8 \epsilon^{2}} u^{d-1} d u
\end{aligned}
$$

Integrating by parts,

$$
\begin{aligned}
& \epsilon A_{d} \int_{0}^{\infty} \frac{f(u)-g(u)}{\{\epsilon+g(u)\}^{2}} u^{d-1} d u \\
& =-2 \epsilon(\alpha+d) \bar{C} \int_{0}^{\infty} \frac{T_{f}(u)-T_{g}(u)}{\left(\epsilon+\bar{C} u^{-\alpha-d}\right)^{2}} u^{-\alpha-d-1} d u .
\end{aligned}
$$

By (3) and the definition of $T_{g}$, there exists a bounded, monotonically decreasing function $M$ on $[0, \infty)$ such that $\lim _{u \rightarrow \infty} M(u)=0$ and $\mid T_{f}(u)-$ $T_{G}(u) \mid \leq M(u) u^{-\alpha}$ for all $u>0$. We want to show that (A.4) is asymptotically negligible relative to $V_{\epsilon}(g) \asymp \epsilon^{\alpha /(\alpha+d)}$ as $\epsilon \downarrow 0$. This result follows from

$$
\begin{aligned}
& \epsilon \int_{0}^{\infty} \frac{\left|T_{f}(u)-T_{g}(u)\right|}{\left(\epsilon+\bar{C} u^{-\alpha-d}\right)^{2}} u^{-\alpha-d-1} d u \\
& \leq \epsilon \int_{0}^{u_{\epsilon}^{1 / 2}} \frac{M(0) u^{-\alpha}}{\left(\bar{C} u^{-\alpha-d}\right)^{2}} u^{-\alpha-d-1} d u+\epsilon \int_{u_{\epsilon}^{1 / 2}}^{u_{\epsilon}} \frac{M\left(u_{\epsilon}^{1 / 2}\right) u^{-\alpha}}{\left(\bar{C} u^{-\alpha-d}\right)^{2}} u^{-\alpha-d-1} d u \\
& \quad+\epsilon \int_{u_{\epsilon}}^{\infty} \frac{M\left(u_{\epsilon}\right) u^{-\alpha}}{\epsilon^{2}} u^{-\alpha-d-1} d u \\
& \leq \frac{\epsilon M(0)}{\bar{C}^{2} d} u_{\epsilon}^{d / 2}+\frac{\epsilon M\left(u_{\epsilon}^{1 / 2}\right)}{\bar{C}^{2} d} u_{\epsilon}^{d}+\frac{M\left(u_{\epsilon}\right)}{\epsilon(2 \alpha+d)} u_{\epsilon}^{-2 \alpha-d},
\end{aligned}
$$

which can be shown to be $o\left(\epsilon^{\alpha /(\alpha+d)}\right)$ using $u_{\epsilon} \asymp \epsilon^{-1 /(\alpha+d)}$. The proposition then follows from (12), (14) and (A.3). 
Proof of Proposition 3: It follows from (16) and $\rho(u)=\int_{0}^{u} \rho^{\prime}(v) d v \geq$ $\int_{0}^{u} v \rho^{\prime}(v) d v / u$ that $\rho(u) \rightarrow \infty$ as $u \rightarrow \infty$. Thus, $u_{j}=\rho^{-1}(j)$ is defined for all $j, 0=u_{0}<u_{1}<\ldots$ and $u_{j} \rightarrow \infty$ as $j \rightarrow \infty$. We can then pick a nonnegative, nondecreasing integer-valued function $L(\epsilon)$ satisfying $L(\epsilon) \rightarrow \infty$ and $\epsilon u_{L(\epsilon)}^{\alpha+d} \rightarrow 0$ as $\epsilon \downarrow 0$. First show that

$$
\epsilon \int_{0}^{\infty}\left\{\frac{f(u)}{\epsilon+f(u)}-\frac{P(\rho(u)) u^{-\alpha-d}}{\epsilon+P(\rho(u)) u^{-\alpha-d}}\right\} u^{d-1} d u=o\left(\epsilon^{\alpha /(\alpha+d)}\right),
$$

which can be done by splitting up the range of integration into, say, $\left[0, u_{L(\epsilon)}\right)$ and $\left[u_{L(\epsilon)}, \infty\right)$ and applying (15) to the second interval to show the integral is sufficiently small as $\epsilon \downarrow 0$. Consider

$$
\begin{aligned}
I_{\epsilon} & =\int_{0}^{\infty} \frac{P(\rho(u)) u^{-\alpha-d}}{\epsilon+P(\rho(u)) u^{-\alpha-d}} u^{d-1} d u \\
& =\int_{0}^{\infty} \frac{P(\rho(u)) u^{d-1}}{\epsilon u^{\alpha+d}+P(\rho(u))} d u \\
& =\sum_{j=0}^{\infty} \int_{u_{j}}^{u_{j+1}} \frac{P(\rho(u)) u^{d-1}}{\epsilon u^{\alpha+d}+P(\rho(u))} d u .
\end{aligned}
$$

It follows from the definition of $L(\epsilon)$ that the terms for $j<L(\epsilon)$ contribute asymptotically negligibly to the sum in the last line. For terms $j \geq L(\epsilon)$, (16) implies

$$
\lim _{\epsilon \downarrow 0} \sup _{j \geq L(\epsilon)} \frac{u_{j+1}}{u_{j}}=1
$$

so

$$
I_{\epsilon} \sim \sum_{j=L(\epsilon)}^{\infty} u_{j}^{d-1} \int_{u_{j}}^{u_{j+1}} \frac{P(\rho(u))}{\epsilon u_{j}^{\alpha+d}+P(\rho(u))} d u .
$$

Writing $\tau$ for $\rho^{-1}$ and making the change of variables $v=\rho(u)$ yields

$$
I_{\epsilon} \sim \sum_{j=L(\epsilon)}^{\infty} u_{j}^{d-1} \int_{j}^{j+1} \frac{P(v)}{\epsilon u_{j}^{\alpha+d}+P(v)} \tau^{\prime}(v) d v .
$$

Now,

$$
\frac{\sup _{j \leq v \leq j+1} \tau^{\prime}(v)}{\inf _{j \leq v \leq j+1} \tau^{\prime}(v)}=\frac{\sup _{u_{j} \leq u \leq u_{j+1}} \rho^{\prime}(u)}{\inf _{u_{j} \leq u \leq u_{j+1}} \rho^{\prime}(u)}
$$


which tends to 1 as $j \rightarrow \infty$ by (17). Therefore, replacing $\tau^{\prime}(v)$ by $\tau(j+1)-$ $\tau(j)=u_{j+1}-u_{j}$ in (A.6) leads to an asymptotically negligible change in the right hand side, so

$$
\begin{aligned}
I_{\epsilon} & \sim \sum_{j=L(\epsilon)}^{\infty} u_{j}^{d-1}\left(u_{j+1}-u_{j}\right) \Phi\left(\epsilon u_{j}^{\alpha+d}\right) \\
& =\epsilon^{-d /(\alpha+d)} \sum_{j=L(\epsilon)}^{\infty}\left(\epsilon^{1 /(\alpha+d)} u_{j}\right)^{d-1} \epsilon^{1 /(\alpha+d)}\left(u_{j+1}-u_{j}\right) \Phi\left(\left(\epsilon^{1 /(\alpha+d)} u_{j}\right)^{\alpha+d}\right),
\end{aligned}
$$

where

$$
\Phi(t)=\int_{0}^{1} \frac{P(v)}{t+P(v)} d v
$$

using $P$ has period 1. Ignoring the factor of $\epsilon^{-d /(\alpha+d)}$ out front, the last line in (A.7) looks like a Riemann sum, so we might expect

$$
\begin{aligned}
\lim _{\epsilon \downarrow 0} \mid & \sum_{j=L(\epsilon)}^{\infty}\left(\epsilon^{1 /(\alpha+d)} u_{j}\right)^{d-1} \epsilon^{1 /(\alpha+d)}\left(u_{j+1}-u_{j}\right) \Phi\left(\left(\epsilon^{1 /(\alpha+d)} u_{j}\right)^{\alpha+d}\right) \\
& -\int_{\epsilon^{1 /(\alpha+d)} u_{L(\epsilon)}}^{\infty} t^{d-1} \Phi\left(t^{\alpha+d}\right) d t \mid=0
\end{aligned}
$$

Note that $\Phi(t) \leq \min (1, \bar{C} / t)$ on $[0, \infty)$, so that $t^{d-1} \Phi\left(t^{\alpha+d}\right)$ is integrable on $[0, \infty)$. If (A.8) holds, then the proposition follows by

$$
\begin{aligned}
\int_{\epsilon^{1 /(\alpha+d)} u_{L(\epsilon)} t^{d-1} \Phi\left(t^{\alpha+d}\right) d t}^{\infty} & \sim \int_{0}^{\infty} t^{d-1} \Phi\left(t^{\alpha+d}\right) d t \\
& =\int_{0}^{1} P(v) \int_{0}^{\infty} \frac{t^{d-1}}{t^{\alpha+d}+P(v)} d t d v \\
& =\frac{1}{\alpha+d} B\left(\frac{d}{\alpha+d}, \frac{\alpha}{\alpha+d}\right) \int_{0}^{1} P(v)^{d /(\alpha+d)} d v
\end{aligned}
$$

where the first step uses $\epsilon^{1 /(\alpha+d)} u_{L(\epsilon)} \rightarrow 0$ as $\epsilon \downarrow 0$, the next step the definition of $\Phi$ and a change in the order of integration and the last step 3.241.4 in Gradshteyn and Ryzhik (2007). 
To prove (A.8), first note

$$
\begin{aligned}
& \mid \sum_{j=L(\epsilon)}^{\infty}\left(\epsilon^{1 /(\alpha+d)} u_{j}\right)^{d-1} \epsilon^{1 /(\alpha+d)}\left(u_{j+1}-u_{j}\right) \Phi\left(\left(\epsilon^{1 /(\alpha+d)} u_{j}\right)^{\alpha+d}\right) \\
& \quad-\int_{\epsilon^{1 /(\alpha+d)} u_{L(\epsilon)}}^{\infty} t^{d-1} \Phi\left(t^{\alpha+d}\right) d t \mid \\
& \leq \sum_{j=L(\epsilon)}^{\infty} \mid\left(\epsilon^{1 /(\alpha+d)} u_{j}\right)^{d-1} \epsilon^{1 /(\alpha+d)}\left(u_{j+1}-u_{j}\right) \Phi\left(\left(\epsilon^{1 /(\alpha+d)} u_{j}\right)^{\alpha+d}\right) \\
& -\int_{\epsilon^{1 /(\alpha+d)} u_{j}}^{\epsilon^{1 /(\alpha+d)} u_{j+1}} t^{d-1} \Phi\left(t^{\alpha+d}\right) d t \mid .
\end{aligned}
$$

Since $\Phi$ is decreasing, on the interval $\left[\epsilon^{1 /(\alpha+d)} u_{j}, \epsilon^{1 /(\alpha+d)} u_{j+1}\right], t^{d-1} \Phi\left(t^{\alpha+d}\right)$ is bounded below by $\epsilon^{(d-1) /(\alpha+d)} u_{j}^{d-1} \Phi\left(\epsilon u_{j+1}^{\alpha+d}\right)$ and above by $\epsilon^{(d-1) /(\alpha+d)} u_{j+1}^{d-1} \Phi\left(\epsilon u_{j}^{\alpha+d}\right)$, so that

$$
\begin{aligned}
& \mid\left(\epsilon^{1 /(\alpha+d)} u_{j}\right)^{d-1} \epsilon^{1 /(\alpha+d)}\left(u_{j+1}-u_{j}\right) \Phi\left(\left(\epsilon^{1 /(\alpha+d)} u_{j}\right)^{\alpha+d}\right) \\
& -\int_{\epsilon^{1 /(\alpha+d)} u_{j}}^{\epsilon^{1 /(\alpha+d)} u_{j+1}} t^{d-1} \Phi\left(t^{\alpha+d}\right) d t \mid \\
\leq & \epsilon^{1 /(\alpha+d)}\left(u_{j+1}-u_{j}\right)\left\{\epsilon^{(d-1) /(\alpha+d)} u_{j+1}^{d-1} \Phi\left(\epsilon u_{j}^{\alpha+d}\right)-\epsilon^{(d-1) /(\alpha+d)} u_{j}^{d-1} \Phi\left(\epsilon u_{j+1}^{\alpha+d}\right)\right\} \\
\leq & \epsilon^{d /(\alpha+d)}\left(u_{j+1}-u_{j}\right)\left[u_{j+1}^{d-1}\left\{\Phi\left(\epsilon u_{j}^{\alpha+d}\right)-\Phi\left(\epsilon u_{j+1}^{\alpha+d}\right)\right\}+\Phi\left(\epsilon u_{j+1}^{\alpha+d}\right)\left(u_{j+1}^{d-1}-u_{j}^{d-1}\right)\right] .
\end{aligned}
$$

For $\eta>0$, by (A.5), we can choose $\epsilon_{1}$ such that for all $j \geq L\left(\epsilon_{1}\right), u_{j+1}^{d-1}-u_{j}^{d-1}<$ $\eta u_{j}^{d-1}$. Furthermore, for $s>t>0$,

$$
\begin{aligned}
\Phi(t)-\Phi(s) & =\int_{0}^{1} \frac{(s-t) P(v)}{(t+P(v))(s+P(v))} d v \\
& \leq \frac{s-t}{t} \int_{0}^{1} \frac{P(v)}{s+P(v)} d v=\frac{s-t}{t} \Phi(s),
\end{aligned}
$$

so that we can pick $\epsilon_{2}$ such that for all $j \geq L\left(\epsilon_{2}\right), \Phi\left(\epsilon u_{j}^{\alpha+d}\right)-\Phi\left(\epsilon u_{j+1}^{\alpha+d}\right) \leq$ $\eta \Phi\left(\epsilon u_{j}^{\alpha+d}\right)$. Finally, we can pick $\epsilon_{3}$ such that for all $j \geq L\left(\epsilon_{3}\right),\left(u_{j+1} / u_{j}\right)^{d-1}<$ 
2. Setting $\epsilon_{0}=\min \left(\epsilon_{1}, \epsilon_{2}, \epsilon_{3}\right)$, we then have for all $j \geq L\left(\epsilon_{0}\right)$,

$$
\begin{aligned}
& \mid\left(\epsilon^{1 /(\alpha+d)} u_{j}\right)^{d-1} \epsilon^{1 /(\alpha+d)}\left(u_{j+1}-u_{j}\right) \Phi\left(\left(\epsilon^{1 /(\alpha+d)} u_{j}\right)^{\alpha+d}\right) \\
& -\int_{\epsilon^{1 /(\alpha+d)} u_{j}}^{\epsilon^{1 /(\alpha+d)} u_{j+1}} t^{d-1} \Phi\left(t^{\alpha+d}\right) d t \mid \\
\leq & \eta \epsilon^{d /(\alpha+d)}\left(u_{j+1}-u_{j}\right)\left\{2 u_{j}^{d-1} \Phi\left(\epsilon u_{j+1}^{\alpha+d}\right)+u_{j}^{d-1} \Phi\left(\epsilon u_{j+1}^{\alpha+d}\right)\right\} \\
\leq & 3 \eta \int_{\epsilon^{1 /(\alpha+d)} u_{j}}^{\epsilon^{1 /(\alpha+d)} u_{j+1}} t^{d-1} \Phi\left(t^{\alpha+d}\right) d t
\end{aligned}
$$

so that for $\epsilon \geq \epsilon_{0}$,

$$
\begin{aligned}
& \mid \sum_{j=L(\epsilon)}^{\infty}\left(\epsilon^{1 /(\alpha+d)} u_{j}\right)^{d-1} \epsilon^{1 /(\alpha+d)}\left(u_{j+1}-u_{j}\right) \Phi\left(\left(\epsilon^{1 /(\alpha+d)} u_{j}\right)^{\alpha+d}\right) \\
& -\int_{\epsilon^{1 /(\alpha+d)} u_{L(\epsilon)}}^{\infty} t^{d-1} \Phi\left(t^{\alpha+d}\right) d t \mid \\
\leq & 3 \eta \int_{\epsilon^{1 /(\alpha+d)} u_{L(\epsilon)}}^{\infty} t^{d-1} \Phi\left(t^{\alpha+d}\right) d t \\
\leq & 3 \eta \int_{0}^{\infty} t^{d-1} \Phi\left(t^{\alpha+d}\right) d t .
\end{aligned}
$$

Since $\eta$ is arbitrary, (A.8) and, hence, Proposition 3, holds.

\section{References}

[1] Abedini, M.J., Nasseri, M., Burn, D.H., 2012. The use of a genetic algorithm-based search strategy in geostatistics: application to a set of anisotropic piezometric head data. Computers \& Geosciences 41, 136146.

[2] Armstrong, M., 1998. Basic Linear Geostatistics. Springer, Berlin.

[3] Bingham, N.H., 1972. A Tauberian theorem for integral transforms of Hankel type. Journal of the London Mathematical Society 2, 493-503. 
[4] Brown, L.D., Carter, A.V., Low, M.G., Zhang, C.-H., 2004. Equivalence theory for density estimation, Poisson processes and Gaussian white noise with drift. Annals of Statistics 32, 2074-2097.

[5] Brown, L.D., Low, M.G., (1996). Asymptotic equivalence of nonparametric regression and white noise. Annals of Statistics 24, 2384-2398.

[6] Chan, G., Hall, P., Poskitt, D.S., 1995. Periodogram-based estimators of fractal properties. Annals of Statistics 23, 1684-1711.

[7] Chan, G., Wood, A.T.A., 2000. Increment-based estimators of fractal dimension for two-dimensional surface data. Statistica Sinica 10, 343376 .

[8] Chan, G., Wood, A.T.A., 2004. Estimation of fractal dimension for a class of non-Gaussian stationary processes and fields. Annals of Statistics $32,1222-1260$.

[9] Chilès, J., Delfiner, P., 2012. Geostatistics: Modeling Spatial Uncertainty, second ed. John Wiley, New York.

[10] Constantine, A.G., Hall, P., 1994. Characterizing surface smoothness via estimation of effective fractal dimension. Journal of the Royal Statistical Society: Series B 56, 97-113.

[11] Datta, A., Banerjee, S., Finley, A.O., Gelfand, A.E., Hierarchical nearest-neighbor Gaussian process models for large geostatistical datasets. arXiv:1406.7343v1.

[12] Davies, S., Hall, P., 1999. Fractal analysis of surface roughness by using spatial data. Journal of the Royal Statistical Society: Series B 61, 3-37.

[13] Emery, X., 2009. The kriging update equations and their application to the selection of neighboring data. Computational Geosciences 13, 269280 . 
[14] Emery, X., Peláez, M., 2011. Assessing the accuracy of sequential Gaussian simulation and cosimulation. Computational Geosciences 15, 673689.

[15] Erdelyi, A., 1954. Tables of Integral Transforms, Volume 1. Scanned copies publicly available.

[16] Gneiting, T., Ševčiková, H., Percival, D.B., 2012. Estimators of fractal dimension: assessing the roughness of time series and spatial data. Statistical Science 27, 247-277.

[17] Golubev, G.K., Nussbaum, M., Zhou, H.H., 2010. Asymptotic equivalence of spectral density estimation and Gaussian white noise. Annals of Statistics 38, 181-214.

[18] Gradshteyn, I.S., Ryzhik, I.M., 2007. Table of Integrals, Series, and Products, seventh ed., A. Jeffrey, D. Zwillinger (Eds.). Academic Press, Orlando.

[19] Haslett, J., 1989. Geostatistical neighbourhoods and subset selection. In M. Armstrong (Ed.) Geostatistics, Kluwer Academic Publishers, Dordrecht, pp. 569-577.

[20] Ibragimov I.A., Rozanov, Y.A., 1978. Gaussian Random Processes, trans. A.B. Aries. Springer-Verlag, New York.

[21] Jakeman, E., Jordan, D.L., 1990. Statistical accuracy of measurements on Gaussian random fractals. Journal of Physics D: Applied Physics 23, 397-405.

[22] Journel, A.G., Huijbregts, Ch.J., 1978. Mining Geostatistics. Academic Press, London.

[23] Kent, J.T., Wood, A.T.A., 1997. Estimating the fractal dimension of a locally self-similar Gaussian process by using increments. Journal of the Royal Statistical Society: Series B 59, 679-699. 
[24] Lam, T.-K., Loh, W.-L., 2000. Estimating structured correlation matrices in smooth Gaussian random field models. Annals of Statistics 28, 880-904.

[25] Olver, F.W.J., Lozier, D.W., Boisvert, R.F., Clark, C.W., 2010. NIST Handbook of Mathematical Functions. Cambridge University Press, Cambridge.

[26] Pinsker, M.S., 1980. Optimal filtering of square integrable signals in Gaussian white noise. Problems of Information Transmission 16, 120133. English translation.

[27] Rivoirard, J., 1987. Two key parameters when choosing the kriging neighborhood. Mathematical Geology 19, 851-856.

[28] Shumway, R.H., Stoffer, D.S., 2011. Time Series Analysis and Its Applications With R Examples, third ed. Springer, New York.

[29] Stein, M.L., 1999. Interpolation of Spatial Data: Some Theory for Kriging. Springer, New York.

[30] Stein, M.L., 2002. The screening effect in kriging. Annals of Statistics 30, 298-323.

[31] Stein, M.L., 2005. Space-time covariance functions. Journal of the American Statistical Association 100, 310-321.

[32] Stein, M.L., 2011. When does the screening effect hold? Annals of Statistics 39, 2795-2819.

[33] Stein, M.L., Chi, Z., Welty, L.J., 2004. Approximating likelihoods for large spatial datasets. Journal of the Royal Statistical Society: Series B 66, 275-296.

[34] Stein, M.L., Handcock, M.S., 1989. Some asymptotic properties of kriging when the covariance function is misspecified. Mathematical Geology 21, 171-190. 
[35] Vecchia, A.V., 1988. Estimation and identification for continuous spatial processes. Journal of the Royal Statistical Society: Series B 50, 297-312.

[36] Wiener, N., 1949. Extrapolation, Interpolation, and Smoothing of Stationary Time Series. MIT Press, Cambridge, MA.

[37] Wu, W., Lim, C., Xiao, Y., 2013. Tail estimation of the spectral density for a stationary Gaussian random field. Journal of Multivariate Analysis $116,74-91$.

[38] Yaglom, A.M., 1987. Correlation Theory of Stationary and Related Random Functions, Volume I: Basic Results. Springer-Verlag, New York. 


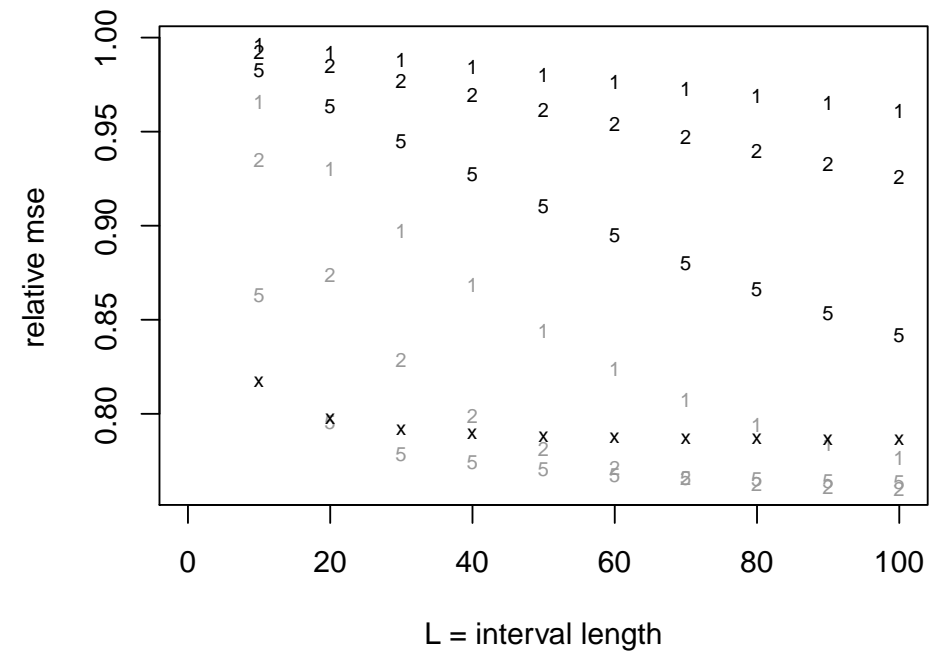

Figure 1 


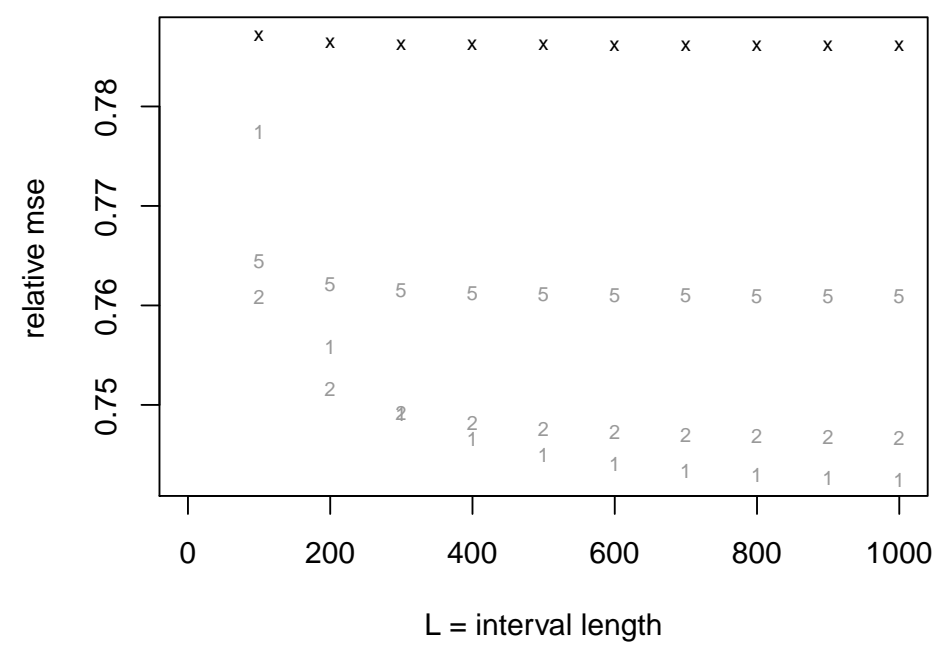

Figure 2 
Figure captions

Figure 1. For the spectral density $h$ in (6), ratios of mean squared errors of optimal predictors of $Z(0)$ based on observations at $\epsilon j$ for $j=1, \ldots, J$ to mean squared error of optimal predictor of $Z(0)$ based on the single observation $Z(\epsilon)$. Values for $J$ given by $J=L / \epsilon$ for $L=10,20, \ldots, 100$, so that $L$ gives the distance from 0 to the most distant observation. Symbols give the value of $\epsilon$ : a black integer $k$ corresponds to $\epsilon=0.001 k$, a gray integer to $\epsilon=0.01 k$ and $\times$ to $\epsilon=0.1$. Thus, when $\delta=0.001$ (the black 1's), $L=100$ corresponds to 100,000 observations.

Figure 2. Same setup as in Figure 1 but for larger values of $L$ and only considering larger $\epsilon$ values. Symbols give the value of $\epsilon$ : a gray integer $k$ corresponds to $\epsilon=0.01 k$ and $\times$ to $\epsilon=0.1$. 